Forum 2020 - 35:363-365

https://doi.org/10.1007/s12312-020-00858-0

Online publiziert: 1. Oktober 2020

C) Springer Medizin Verlag $\mathrm{GmbH}$, ein Teil von Springer Nature 2020

Deutsche Krebsgesellschaft e.V.

Berlin, Deutschland

\section{Nutzung von Registerdaten für Forschung, Versorgung und Nutzenbewertung. Was ist möglich, was nicht?}

\author{
Kurzbericht zum „Brennpunkt Onkologie“ \\ vom 08.09.2020
}

Können in Registern erfasste Daten aus der Versorgung Wissenslücken in der Onkologie schließen? Auf dem „Brennpunkt Onkologie“ am 08.09.2020 diskutierten hierzu Vertreter der Deutschen Krebsgesellschaft sowie des Instituts für Qualität und Wirtschaftlichkeit im Gesundheitswesen (IQWiG) und forderten ein zentrales Spitzeninstitut für Versorgungsforschung.

Patient*innen mit seltenen oder schwer lebensbedrohlichen Erkrankungen müssen schnellstmöglich von neuen Therapien profitieren - zugleich sollen die Arzneimittel aber im Vergleich zu bereits etablierten Therapien einen Mehrwert haben. Mit der Zulassung von Orphan Drugs wird genau dieses Ziel verfolgt. Die Realität zeigt allerdings: Die Datenlage zum Markteintritt ist häufig begrenzt. Es fehlen Erhebungen zur Mortalität, Morbidität, Nebenwirkungen und Lebensqualität. Aber wie kann diese Evidenzlücke geschlossen werden? Dr. Thomas Kaiser, Ressortleiter Arzneimittelbewertung im IQWiG, sprach auf dem „Brennpunkt Onkologie“ über die Möglichkeit, versorgungsnahe Daten für Therapieentscheidungen zu nutzen (• Abb. 1). Das IQWiG legte zu Jahresanfang mit einem „Rapid Report" eine wissenschaftliche Ausarbeitung zu den Qualitätsanforderungen versorgungsnaher Daten vor. Hierfür analysierte das IQWiG Orphan Drugs, die der Gemeinsame Bundesausschuss zwischen 2014 und 2018 bewertet hatte. Fazit: „Bis- lang war die Quantifizierung des Zusatznutzens nur möglich, wenn RCTs vorlagen. Aber auch von den vorgelegten RCTs waren nur etwa $40 \%$ dafür geeignet. Außerdem fehlten regelhaft Daten zur Kontrollgruppe, und Evidenzlücken zeigten sich in allen Endpunkten“, so Kaiser. Unter Einhaltung bestimmter Qualitätsanforderungen könnten Registerdaten hier einen Erkenntnisgewinn liefern. Hierfür gibt es aber noch weiteren Handlungsbedarf - unter anderem bei dem The-

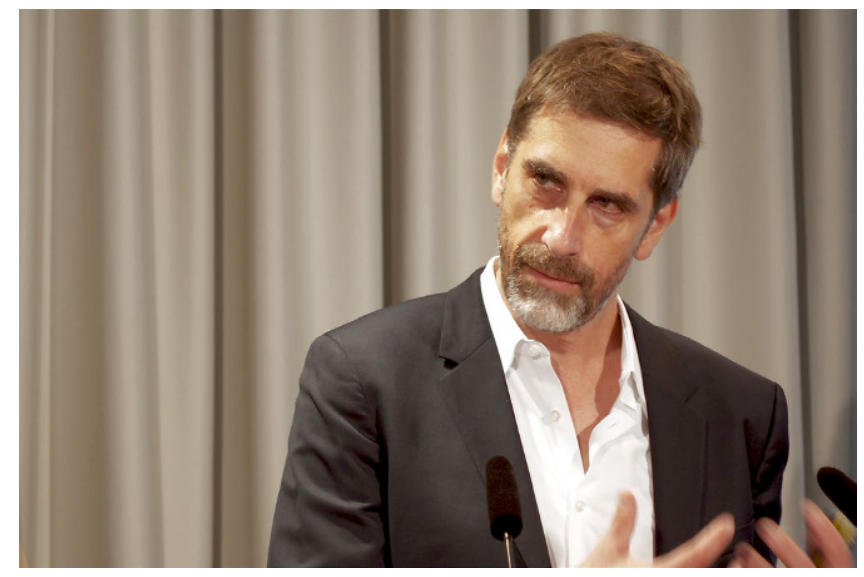

Abb. $1<$ Dr. Thomas Kaiser. Institut für Qualität und Wirtschaftlichkeit im Gesundheitswesen (IQWiG), Köln. (@ Renate Babnik/ DKG)

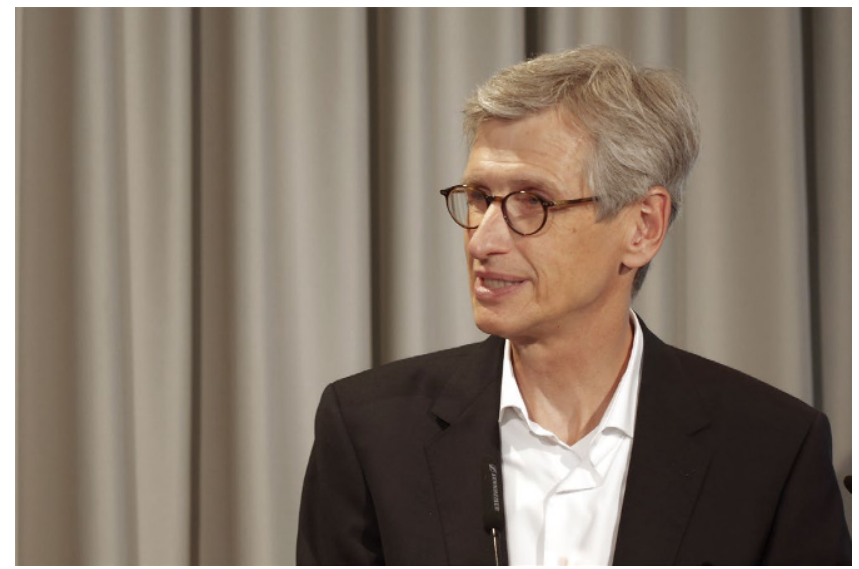

Abb. $2 \triangleleft$ Prof. Dr. Thomas Seufferlein. Präsident der Deutschen Krebsgesellschaft. Berlin (@ Renate Babnik/ DKG) 
ma Datenschutz und standardisierte ITAnwendungen. Diese variieren nämlich je nach Bundesland, beziehungsweise je nach Klinik, was eine hohe Hürde für den Aufbau qualitativ hochwertiger Register darstellt. Auch eine Finanzierung der Register müsse auf sichere Beine gestellt werden, sagte Kaiser. DKG-Präsdident Professor Dr. Thomas Seufferlein verdeutlichte in seinem Vortrag, welchen Nutzen versorgungsnahe Daten für die Forschung, Versorgung und Leitlinienentwicklung haben können (• Abb. 2). Als Beispiele aus der Onkologie zog er die klinischen Krebsregister und die zertifizierten Zentren der DKG mit dem Projekt Oncobox Research heran. Anhand der Zentren lassen sich bereits versorgungsnahe Daten im Rahmen verschiedener Projekte nutzen und valide Informationen für Therapie und Strukturempfehlungen - etwa zu Mindestmengen - bereitstellen. Für die Leitlinienentwicklung könnten anhand von versorgungsnahen Daten etwa Effekte von Therapiedurchführungen in der realen Versorgung untersucht werden. Voraussetzung hierfür sei aber die Vollständigkeit der Daten, die Qualitätssicherung und -prüfung und nicht zuletzt eine klare klinische Fragestellung, so Seufferlein. Für ihn ist die Versorgungsforschung in der Onkologie ein hochkomplexes Forschungsfeld: „Versorgungsforschung ist Spitzenforschung, deshalb benötigen wir ein Spitzeninstitut für onkologische Versorgungsforschung."

Auf der anschließenden Podiumsdiskussion sprach Dr. Johannes Bruns, Generalsekretär der DKG, zusammen mit Kaiser und Seufferlein über die Möglichkeiten und Grenzen der Nutzung von Registerdaten. Registerstudien können keine klinischen Studien ersetzen, aber sinnvoll ergänzen - das war einerseits der Tenor. Auf der anderen Seite wurde aber auch klar, dass Registerstudien auch RCTs sein können. Gerade mit registerbasierten RCTs könne aus Sicht von Kaiser das Beste aus zwei Welten zusammengebracht werden. „Wir sprechen bei Registern über Datenqualität. Aber wie stellen wir sicher, dass die im Versorgungsalltag von Ärzten erhobenen Daten qualitativ hochwertig sind?“, so Bruns. Die Politik sollte stärkere Anreize für eine gute Datenerhebung schaffen - sei es finanziell oder aber, um die eigene Qualität in der klinischen Praxis zu verbessern, so Bruns. In der Diskussion sprachen sich die Referenten gemeinsam für eine zentrale Stelle zur Versorgungsforschung aus, die auch übergeordnete klinische Fragestellungen definiert und die Forschung methodisch begleitet, um eine hochwertige Versorgungsforschung zu gewährleisten.

Der „Brennpunkt Onkologie“ fand unter strengen Hygienevorschriften mit etwa 60 Gästen in der Kalkscheune in Berlin statt. Moderiert wurde die Veranstaltung von Lisa Braun, Presseagentur Gesundheit.

\section{Mehr Infos}

Auf der DKG-Webseite finden Sie unter diesem Link https://tinyurl.com/versorgungsnahe-daten

- die Präsentationen der Referenten, sofern deren Einwilligung zur Veröffentlichung vorliegt,

- zeitnah einen ausführlichen Nachbericht, denn die Veranstaltung wird dokumentiert,

- die Fragen aus dem digitalen Vorzimmer

\section{Nächster Brennpunkt Onkologie}

Wir freuen uns auf Ihre Teilnahme und Ihrer Diskussionsfreude am

- Di, 17. November 2020

- 12.30 Uhr bis 15.30 Uhr

- Kalkscheune Berlin

www.krebsgesellschaft.de/brennpunkt.html

\section{Korrespondenzadresse}

Deutsche Krebsgesellschaft e.V.

Kuno-Fischer-Straße 8, 14057 Berlin,

Deutschland

service@krebsgesellschaft.de

www.krebsgesellschaft.de

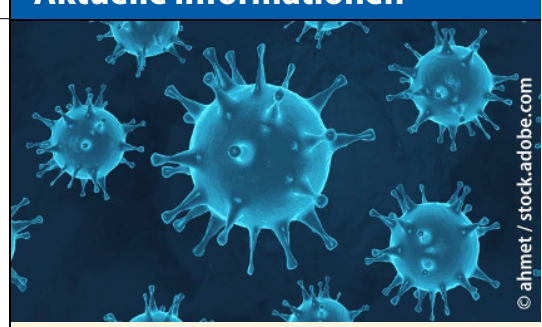

\section{Aktuelle Entwicklung zu COVID-19 bei Springer Nature und Springer Medizin}

Springer Nature und Springer Medizin unterstützen die globale Reaktion auf die COVID-19-Pandemie, indem ein schneller und direkter $\mathrm{Zu}$ gang zu den neuesten verfügbaren Forschungsergebnissen und Daten ermöglicht wird.

Auf der Homepage SpringerMedizin.de finden Sie ein immer aktuelles Dossier mit Beiträgen, Forschungsarbeiten und Ergebnissen zu SARS-CoV-2 sowie relevanten Links.

Darin z.B. auch die kürzlich publizierte

Empfehlung von DIVI, DGIIN, DGAI und DGP zur Intensivtherapie von Patienten mit COVID-19.

Springer Nature arbeitet mit globalen Organisationen zusammen, und verlinkt über SpringerNature.com/de auf eine eigene Landingpage mit einer Vielzahl an Information sowie freiem Zugriff auf die COVID-19-Contentplattformen von Nature Research, BioMed Central (BMC) und Springer.

Das Dossier zu Coronavirus / Covid-19 von Springer Medizin finden Sie hier: www.springermedizin.de/covid-19

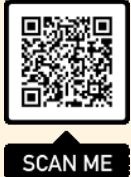


Hier steht eine Anzeige.

\section{算 Springer}

